\title{
An Iranian Version of the Fukushima Lumbar Spinal Stenosis Scale (FLS-25): A Validation Study
}

\author{
Parisa Azimi ${ }^{1}$, Ali Montazeri ${ }^{2}$ \\ ${ }^{1}$ Functional Neurosurgery Research Center, Shahid Beheshti University of Medical Sciences, Tehran, Iran \\ ${ }^{2}$ Mental Health Research Group, Health Metrics Research Centre, Iranian Institute for Health Sciences Research, ACECR, Tehran, Iran
}

\begin{abstract}
Study Design: Cross-sectional study.
Purpose: To translate and validate the Fukushima lumbar spinal stenosis (LSS) scale 25 (FLS-25) for use in Iran.

Overview of Literature: Tools measuring patient-reported outcomes should satisfy certain psychometric properties.

Methods: FLS-25 is a self-administered scale for evaluating symptoms of LSS. A forward-backward procedure was applied to translate the questionnaire from English into Persian. A sample of patients with LSS completed the questionnaire at two points in time: once before surgery and once 6 months after the surgery. The Neurogenic Claudication Outcome Score (NCOS) was also used for assessment. The psychometric properties of FLS-25 were evaluated for internal consistency, test-retest and interobserver reliabilities, responsiveness to change, known-group comparison, and convergent validity.

Results: In all, 131 patients were included in the study. The mean age of the patients was 61.4 (standard deviation, 11.1) years. The Cronbach's alpha coefficient for FLS- 25 was 0.89 . Test-retest reliability as carried out by the intraclass correlation coefficient was 0.94 (95\% confidence interval, 0.95$)$. Interobserver agreement as measured by the kappa statistics also was found to be acceptable (kappa value, 0.88 ), and validity was found to be satisfactory. The instrument was able to discriminate between the subgroups of patients who differed in symptom severity. The correlation between FLS-25 and NCOS scores was excellent, indicating good convergent validity $(r=0.82, p<0.001)$. The results also indicated that the instrument was responsive to change $(p<0.001)$.

Conclusions: The Iranian version of FLS-25 performed well, and the findings suggest that it is a valid measure of symptom severity in LSS patients.
\end{abstract}

Keywords: FLS-25; Validity; Iran; Lumbar spinal stenosis

\section{Introduction}

Lumbar canal stenosis (LSS) is frequently diagnosed in cases of lower back pain and is caused by degeneration of the spinal components, including the intervertebral disc, facet, and ligamentum flavum [1]. Symptoms of LSS include neurogenic claudication, pain, heaviness, numb- ness, and cramping in the lower limb [2]. These symptoms usually improve with appropriate conservative treatment; however, depending on symptom severity and functioning, surgical intervention is occasionally needed [3]. Therefore, it is important to measure symptom severity and functioning in LSS patients. By determining the cutoff values of an instrument used to assess LSS symptoms,

\footnotetext{
Received Jun 4, 2016; Revised Jul 5, 2016; Accepted Jul 24, 2016

Corresponding author: Parisa Azimi

Functional Neurosurgery Research Center of Shohada Tajrish Hospital, Shahid Beheshti University of Medical Sciences,

Sharadari St., Tajrish Square, Tehran 1989934148, Iran

Tel:98-2122749204, Fax: +98-2188265188, E-mail: parisa.azimi@gmail.com
} 
such a tool may also be applied to the clinical decisionmaking process.

Various questionnaires as the Oswestry disability index, Swiss Spinal Stenosis Questionnaire, and Oxford Claudication Score have been used to evaluate symptom severity and functioning in LSS patients. However, these questionnaires do not comprehensively measure specific LSS symptoms and they do not evaluate a wide range of treatments; the LSS-specific symptom scale (Fukushima LSS scale 25, FLS-25) was developed for this purpose [4-6]. FLS-25 is a disease-specific tool that measures the severity of symptoms in LSS, and it is simple and self-administered [4]. Several studies have reported on the psychometric properties of FLS-25 [4-6]. The aim of this study was to translate FLS-25 into Persian (Iranian language) and validate the use of the questionnaire in LSS outcome studies conducted with Iranian patients.

\section{Materials and Methods}

\section{Translation}

FLS-25 was translated into Persian from English by two independent academics unconnected to the study. Consequently, a consolidated forward translation was drafted; then, this was backward translated into English by two other academics. After providing a consolidated backward questionnaire, the pre-final Persian version of the questionnaire was ready for pilot testing to establish that it would be understood and that the questions measured what they were intended to measure.

\section{The questionnaire}

Symptoms of LSS are measured using a specific LSS symptom scale (FLS-25). It was designed by Sekiguchi et al. [4-6] and asks patients about their symptoms in specific situations. It consists of 25 questions and all items are rated on a 4-point Likert scale. Possible scores for the items range from 0 (strongly disagree) to 4 (strongly agree). Overall scores on the questionnaire range from 0 to 100, with higher scores indicating better conditions (Appendix 1).

\section{Patients and data collection}

The final draft of the Iranian version was administered to a sample of newly diagnosed LSS patients who underwent surgery at the neurosurgery clinic of a teaching hospital in Tehran, Iran between April 2012 and June 2014. LSS diagnosis was confirmed on the basis of clinical symptoms, neurological examinations, and imaging data, including radiography, computed tomography $(\mathrm{CT})$, and magnetic resonance imaging (MRI) of the lumbar spine. All cases presented with the typical symptoms of LSS, which include neurogenic intermittent claudication and numbness and/or leg pain. In all cases, diagnosis was confirmed by more than one spine surgeon. The level(s) of stenosis were localized using CT or MRI. More than one spine surgeon confirmed the diagnosis. All patients received conservative treatment for at least 6 months. Because all patients presented with the typical symptoms of LSS, they were all candidates for surgery. For each patient, two health professionals trained in neurosurgery independently scored the questionnaire at admission (a day before surgery); both independent observers assessed patients the same day. The observers were unaware of the purpose of the study. Patients were assessed once again at 6 months postoperative follow-up.

Patients received surgery (laminectomy without fusion [ $n=116]$ or with fusion $[n=15]$ at one to three levels, unilaterally or bilaterally, depending on the degree of stenosis) if conservative treatment failed.

\section{Measures}

The severity of LSS based on the morphology of the dural sac was used to classify patients according to stenosis grade. Constantin and colleagues described a 7-grade (A: $A 1, A 2, A 3, A 4, B, C$, and D) classification for the severity of LSS based on the morphology of the dural sac as observed on T2 axial magnetic resonance images using the rootlet/cerebrospinal fluid ratio [7]. They defined grade A as no or minor stenosis, $\mathrm{B}$ as moderate stenosis, $\mathrm{C}$ as severe stenosis, and $\mathrm{D}$ as extreme stenosis [7]. The Iranian version of the Neurogenic Claudication Outcome Score (NCOS) was also used to indicate functionality. NCOS is a specific measure of functionality in patients with neurogenic claudication and consists of 16 items (8 questions). Each item is rated on a four-point scale with a 2-point intervals ranging from 0 to 6 (0-2-4-6), from worst to best conditions; a $100-\mathrm{mm}$ visual analog scale is used for pain intensity. The scale score is then calculated by summation of all 16 items ranging from 0 to 100, with higher scores indicating higher levels of functioning and/or better health status [8]. 


\section{Statistical analysis}

To test reliability, the internal consistency of the questionnaire was calculated using Cronbach's alpha coefficient, and a Cronbach's alpha $\geq 0.70$ was considered satisfactory [9]. In addition, interobserver reliability was assessed using the kappa statistics [9]. We also performed test-retest reliability using the intraclass correlation coefficients (ICCs) to examine stability. Raters completed NCOS twice at a 24-hour interval. An ICC above 0.80 was considered evidence of excellent stability [9].

To measure validity, different procedures were used. Known-groups comparison was performed to assess validity [9] and was applied to examine how well the questionnaire discriminated between subgroups of patients who differed in dural sac morphology based on MRI. It was hypothesized that the preoperative FLS-25 score for grade-C stenosis would be lower than grade-D stenosis. Convergent validity (criterion validity) was performed to determine how well the questionnaire correlates with another valid instrument measuring similar constructs. Accordingly, correlations between FLS-25 and NCOS were calculated. Pearson's correlation coefficients and values of $\geq 0.40$ were considered satisfactory $(r \geq 0.81-1.0$ as excellent; $0.6-0.80$ as very good; $0.41-0.60$ as good; $0.21-0.40$ as fair; $0.0-0.20$ as poor) $[9,10]$. Finally, responsiveness to change as a psychometric property of the questionnaire was also assessed; as such, patients' preoperative and postoperative scores were compared using the paired $t$ test to see if the scale was able to capture changes after surgery.

\section{Ethics}

The Ethics Committee of Shahid Beheshti University of Medical Sciences in Tehran, Iran approved the study.

\section{Results}

The study consisted of 131 LSS patients, including 85 patients with central stenosis and 46 patients with central canal stenosis and lateral canal stenosis. The mean age of the patients was 61.4 (standard deviation, 11.1) years; most were married (75.6\%) and had completed primary or secondary education (64.9\%). The characteristics of patients, including lumbar stenosis levels and their scores on FLS-25 and NCOS, are shown in Table 1. Overall, 186 levels were treated by surgery (Table 1). All patients had
Table 1. Characteristics of the study patients and their scores on the FLS-25 ( $n=131)$

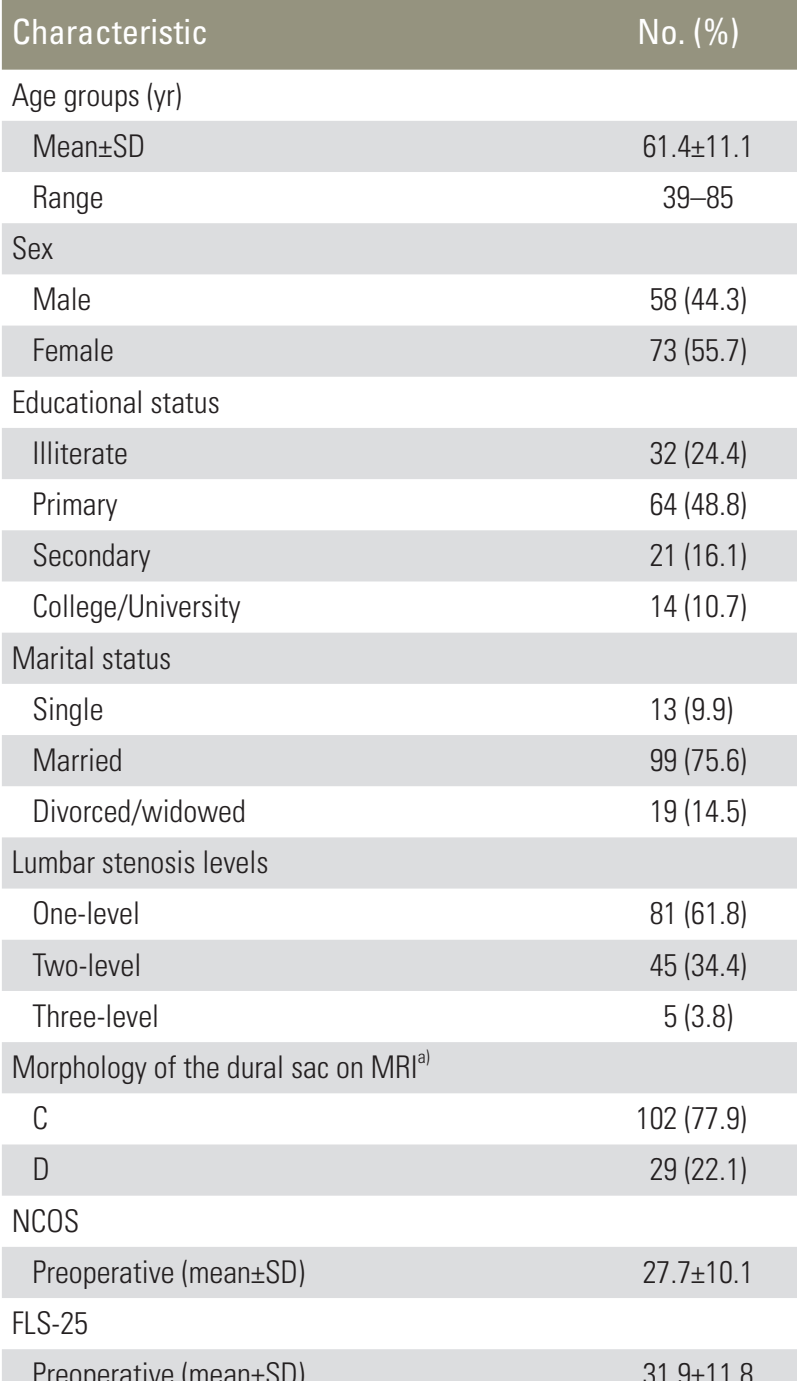

FLS-25, Fukushima lumbar spinal stenosis scale 25; SD, standard deviation; NCOS, Neurogenic Claudication Outcome Score.

${ }^{a}$ Extreme (D) and severity (C) stenosis of lumbar spinal stenosis based on the morphology of the dural sac as observed on T2 axial magnetic resonance imaging (MRI).

Table 2. Known-groups comparison

Variable Preoperative

Morphology of the dural sac on MRI ${ }^{a)}$

\begin{tabular}{lc} 
D & $24.6 \pm 10.7$ \\
C & $34.1 \pm 12.1$ \\
$p$-value $^{\text {b) }}$ & $<0.001$ \\
\hline
\end{tabular}

Values are presented as mean \pm standard deviation.

${ }^{a}$ Extreme (D) and severity (C) stenosis of lumbar spinal stenosis based on the morphology of the dural sac as observed on T2 axial magnetic resonance imaging (MRI). There were no A and B categories; ${ }^{b}$ Derived from $t$-test. 
Table 3. Responsiveness to change

\begin{tabular}{lccc} 
Score & Preoperative & Postoperative & $p$-value ${ }^{\text {a) }}$ \\
FLS-25 score & $31.9 \pm 11.8$ & $72.1 \pm 15.4$ & $<0.001$ \\
\hline
\end{tabular}

Values are presented as mean \pm standard deviation. FLS-25, Fukushima lumbar spinal stenosis scale 25.

a) Derived from $t$-test.

grade- $C(n=102)$ and grade-D ( $n=29)$ stenosis. Statistically significant differences were observed between preoperative and postoperative scores on FLS-25, indicating improvements in outcome and functionality $(p<0.001)$.

The internal consistency of FLS-25 was found to be satisfactory. The Cronbach's alpha coefficient was 0.89 and the agreement between two independent observers was 0.82 , indicating desirable interobserver reliability. Testretest reliability, as carried out by ICC, was found to be 0.94 (95\% confidence interval, 0.96-0.92), reflecting excellent stability for FLS-25. Validity of FLS-25 was examined using the known-groups comparison. FLS-25 well discriminated between subgroups of patients who differed in morphology $(p<0.001)$ (Table 2$)$. In addition, NCOS scores were strongly correlated with patient's preoperative scores on FLS-25 $(r=0.82, p<0.001)$. Responsiveness to change was assessed by paired $t$ test. In all instances, FLS- 25 was able to detect changes after surgery, indicating improvements as expected (Table 3).

\section{Discussion}

Overall, the results of the present study showed that the Persian version of FLS-25 is a reliable and valid tool for measuring LSS symptoms in Persian-speaking patients. Our patients were at ease when responding to the questionnaire. Although the tool is time-consuming, if enough time is spared in the translation process, satisfactory psychometric results may be achieved.

We measured the severity of LSS based on dural sac morphology, as observed on T2 axial MRI, as a clinical measure for known-groups comparison. FLS-25 could discriminate between subgroups of patients who differed in the grade of stenosis during preoperative assessment, as expected.

Sekiguchi et al. [6] reported on the responsiveness of FLS-25 in patients with LSS who received conservative treatment. They showed that FLS-25 has the potential to be used in patients with various types of LSS. The present study showed that FLS-25 can be used to assess the effects of surgical treatment, although this study included only patients who had grade- $\mathrm{C}$ and grade-D stenosis.

Sekiguchi et al. [6] reported that FLS-25 scores are correlated with self-reported walking times, stress test standing times, walking distances, and walking times in a population of patients with confirmed LSS who required conservative treatment. In this study, we also found a strong correlation between FLS-25 and NCOS at preoperative assessment. NCOS was selected for convergent validity because NCOS is a suitable instrument for measuring functionality in patients with LSS [8].

Although instruments such as the Oswestry disability index, Swiss Spinal Stenosis Questionnaire, Oxford Claudication Score, Roland-Morris Disability Questionnaire, SF-36, and EuroQol (EQ5D) are useful for measuring changes that occur after clinical interventions, their sensitivity is limited in determining therapeutic effectiveness for all levels of LSS severity [5]. In fact, these scales are also limited in capturing the intensity and the nature of symptoms associated with LSS [5]. Thus, it has been proposed that FLS-25 was developed to comprehensively measure patient symptoms of LSS instead of evaluating global questions [4-6].

\section{Conclusions}

The Iranian version of FLS-25 performed well, and our findings suggest that it is a valid measure of symptom severity in patients with LSS.

\section{Conflict of Interest}

No potential conflict of interest relevant to this article was reported.

\section{Acknowledgments}

The authors thank the staff of the Neurosurgery Unit 
Imam-Hossain Hospital, Tehran, Iran.

\section{References}

1. Kreiner DS, Shaffer WO, Baisden JL, et al. An evidence-based clinical guideline for the diagnosis and treatment of degenerative lumbar spinal stenosis (update). Spine J 2013;13:734-43.

2. Porter RW. Spinal stenosis and neurogenic claudication. Spine (Phila Pa 1976) 1996;21:2046-52.

3. Omidi-Kashani F, Hasankhani EG, Ashjazadeh A. Lumbar spinal stenosis: who should be fused? An updated review. Asian Spine J 2014;8:521-30.

4. Sekiguchi M, Wakita T, Fukuhara S, et al. Development and validation of a quality of life scale specific for lumbar spinal stenosis. Spine (Phila Pa 1976) 2011;36:E1407-14.

5. Sekiguchi M, Wakita T, Otani K, et al. Development and validation of a symptom scale for lumbar spinal stenosis. Spine (Phila Pa 1976) 2012;37:232-9.
6. Sekiguchi M, Wakita T, Otani K, et al. Lumbar spinal stenosis-specific symptom scale: validity and responsiveness. Spine (Phila Pa 1976) 2014;39:E1388-93.

7. Schizas C, Theumann N, Burn A, et al. Qualitative grading of severity of lumbar spinal stenosis based on the morphology of the dural sac on magnetic resonance images. Spine (Phila Pa 1976) 2010;35:191924.

8. Azimi P, Mohammadi HR, Montazeri A. An outcome measure of functionality in patients with lumber spinal stenosis: a validation study of the Iranian version of Neurogenic Claudication Outcome Score (NCOS). BMC Neurol 2012;12:101.

9. Nunnally JC, Bernstien IH. Psychometric theory. 3rd ed. New York: McGraw-Hill; 1994.

10. Tomkins-Lane CC, Battie MC. Validity and reproducibility of self-report measures of walking capacity in lumbar spinal stenosis. Spine (Phila Pa 1976) 2010; 35:2097-102. 
Appendix 1. Items composing the FLS-25

1) Sometimes numbness or pain interferes with my ability to concentrate on things.

2) I sometimes have leg cramps when I lie down to sleep.

3) I cannot walk unless I bend forward.

4) When I am sleeping, the ends of my feet feel cold.

5) When I stretch my back, my symptoms get worse.

6) Sometimes when I try to walk, I can't move my legs forward and I almost fall.

7) I can't lie on my back.

8) I spend most of the day lying down due to pain or numbness.

9) I can't turn over in bed.

10) I have difficulty walking down stairs.

11) I can only walk slowly.

12) I can't do work or chores where I have to stand in one place.

13) If I stand for a while, I feel numbness or pain and cannot keep standing.

14) When I can't walk any further, I can walk again after resting for a short while.

15) I sometimes stagger when I stand up or sit down.

16) I have difficulty walking up stairs.

17) I can't stretch my back.

18) My symptoms are worst when I wake up in the morning.

19) I often have no feeling in my legs.

20) If I walk for a while, my symptoms worsen and I can't walk anymore.

21) Numbness or pain bothers me even when I'm eating.

22) I can't walk because it makes me feel exhausted.

23) I can't twist my body to look above or behind me.

24) When walking up or down stairs, I must use the handrail.

25) Sitting back up from a lying position requires effort.

Instructions: "The following questions concern symptoms and problems related to the lower limbs. Indicate how much you agree or disagree with the following statements about your daily life by circling a number from 0 (Strongly disagree) to 4 (strongly agree)." Likert choices: $0=$ strongly disagree, $1=$ somewhat disagree, $2=$ neither agree nor disagree, $3=$ somewhat agree, and $4=$ strongly agree.

FLS-25, Fukushima lumbar canal stenosis scale 25. 